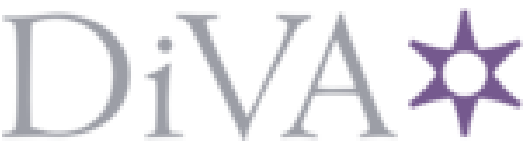

http://www.diva-portal.org

\title{
Postprint
}

This is the accepted version of a paper presented at 9th Swedish Workshop on Data Science, SweDS 2021, Virtual, Vaxjo, 2 December 2021 through 3 December 2021.

Citation for the original published paper:

Lewenhagen, K., Boldt, M., Borg, A., Gerell, M., Dahlen, J. (2021)

An Interdisciplinary Web-based Framework for Data-driven Placement Analysis of CCTV Cameras

In: Proceedings of the 2021 Swedish Workshop on Data Science, SweDS 2021 Institute of Electrical and Electronics Engineers Inc.

https://doi.org/10.1109/SweDS53855.2021.9637719

N.B. When citing this work, cite the original published paper.

(C)2021 IEEE. Personal use of this material is permitted. Permission from IEEE must be obtained for all other uses, in any current or future media, including reprinting/republishing this material for advertising or promotional purposes, creating new collective works, for resale or redistribution to servers or lists, or reuse of any copyrighted component of this work in other works. 


\section{An Interdisciplinary Web-based Framework for Data-driven Placement Analysis of CCTV Cameras}

\author{
Kenneth Lewenhagen, Martin Boldt, Anton Borg \\ Department of Computer Science and Engineering \\ Blekinge Institute of Technology \\ Karlskrona, Sweden \\ \{kenneth.lewenhagen,martin.boldt,anton.borg\}@bth.se
}

\author{
Manne Gerell \\ Department of Criminology \\ Malmö University \\ Malmö, Sweden \\ manne.gerell@mau.se
}

\author{
Johan Dahlén \\ Camera and analysis section \\ Investigations Division, Region South \\ Swedish Police \\ johan-h.dahlen@polisen.se
}

\begin{abstract}
This paper describes work in progress of an interdisciplinary research project that focuses on the placement and analysis of public close-circuit television (CCTV) cameras using data-driven analysis of crime data. A novel web-based prototype that acts as a framework for the camera placement analysis with regards to historical crime occurrence is presented. The webbased prototype enables various analyses involving public CCTV cameras e.g., to determine suitable locations for both stationary CCTV cameras as well as temporary cameras that are moved around after a few months to address crime seasonality. The framework also opens up for other analyses, e.g. automatically highlighting crimes that are carried out closed by at least one camera. The research also investigates to what extent it is possible to generate estimates on the amount of detail captured by a camera given the distance to the crime light conditions. The research project includes interdisciplinary competences from various areas such as criminology, computer and data science as well as the Swedish Police.

Index Terms-CCTV camera placement, data-driven placement analysis, web-based prototype, intelligent models.
\end{abstract}

\section{INTRODUCTION}

Law enforcement agencies are relying on public closecircuit television (CCTV) cameras as an important source for evidence material related to criminal events [1]. This is a reasonable development given the growing body of knowledge that supports that CCTV cameras have some inhibiting effect on crime events, at least at some locations and for some types of crimes, and especially when complemented with additional interventions, e.g. lighting and police patrols [2]. However, there is limited research on the spatial/temporal placement of CCTV cameras in relation to criminal activity. Therefore, this paper describes work in progress that focuses on the placement of public CCTV cameras by means of data-driven analysis of historical crime patterns.

This interdisciplinary research is carried out by Blekinge Institute of Technology, Malmö University, and the Swedish Police. The geographical area considered is the city of Malmö, in the south of Sweden. The research involves the placement of two different categories of cameras. First, stationary public CCTV cameras that are permanently installed in the city. Secondly, temporary public CCTV cameras that are removed after one, two or three months, and thus can be used to address seasonality variations of crimes. In addition to the placement of cameras the project also investigates to what extent it is possible to generate estimates on the amount of detail captured by each camera given the distance to the crime as well as weather and light conditions at the particular time.

This paper describes a novel web-based prototype that acts as a framework for the analysis of camera placement with regards to historical crime occurrence. Using this web-based prototype it is possible for the analyst to detect whether crimes were registered by public CCTV cameras, but also detect crimes that are carried out within some distance from a camera, e.g. 100 meters. It is also possible to search for crimes given various parameters, e.g. different date intervals or particular crime categories that are of interest.

This is the first step towards providing smart camera placement and camera analysis with both a crime investigation perspective, i.e. investigating crimes, and a crime prevention perspective. By enabling the use of smart ICT, the strain on law enforcement resources can be alleviated by automating e.g. manual, routine tasks [3].

\section{A. Related Work}

Although widely used there is surprisingly little research on the use of CCTV footage in crime investigations. However, Ashby investigates some $251^{\prime} 000$ crimes that occurred in the British railway network between 2011 and 2015 [4]. CCTV footage was available in $45 \%$ of those crimes, and was judged to be useful for $65 \%$ of those particular crimes. The results indicate that useful CCTV footage is associated with significantly increased chances of crimes being solved regardless of type of crime.

Gerell investigates changes in crime, and crime clearance, associated with CCTV in the city of Gothenburg, Sweden. The study used analysis of three deprived neighbourhoods as treatment areas, and with three other areas used as controls [5]. The results indicate that crime clearance rates increased for both property crimes and violent crimes in the treatment areas (relative to controls), although no changes were significant.

Further, Jung and Wheeler investigates to what extent public CCTV cameras increase case clearance rates in Dallas, Texas [6]. Their results indicate that crimes committed closer to cameras were associated with an increased clearance rate after the cameras were installed. However, the effects were only local around the CCTV cameras, and were to large extent 


\begin{tabular}{lll}
\hline Name & Type & Description \\
\hline $\begin{array}{l}\text { Name } \\
\text { Geo-position }\end{array}$ & $\begin{array}{l}\text { String } \\
\text { Long/Lat }\end{array}$ & $\begin{array}{l}\text { Name of the camera } \\
\text { Longitude/latitude coordinates of } \\
\text { camera position }\end{array}$ \\
Coverage & $\{$ Long/Lat $\}$ & $\begin{array}{l}\text { A set of Long/Lat positions that } \\
\text { encapsulates the geographical area } \\
\text { covered by the camera }\end{array}$ \\
\hline
\end{tabular}

TABLE I: Columns in camera dataset.

limited to thefts. Further, the authors stress the importance of camera placement in order to get better justification for the use of CCTV cameras.

There are limited research into CCTV placement, with some focusing on policies for local camera placement [7], and others on overlapping coverage [8]. While new technologies can be used to automate detecting crimes from camera images, or identify persons over several cameras [3], it is still important to place cameras correctly [7].

Genetic algorithms has been suggested to aid with camera placement around schools in Korea [7]. Based on Routine Activity Theory and rational choice perspective, the authors use genetic algorithms to explore where cameras should be placed with regard to e.g. visibility. The results indicate that it is possible to identify camera placement with high visibility for streets with both high and low pedestrian traffic.

No prior research that explicitly focuses on the process of public CCTV camera placement over a larger geographic area, e.g. a city, with regards to criminal events has been found. Thus, this is the identified research gap that this study makes a first attempt to address.

\section{B. Aim \& Scope}

The aim of this study is to investigate the aspects involved in the placement of public CCTV cameras, using data-driven crime analyses, with the goal to increase the clearance rates of crimes.

The scope of this study involves the city of Malmö in Sweden. Only public CCTV cameras that are controlled by Swedish law enforcement are included in the camera dataset.

\section{Ethical concerns}

No crime data that can be associated with individual persons are included in the crime dataset used in this study, i.e. residential burglaries and domestic violence crimes were excluded. It should also be noted that this research does not require any access to the actual CCTV camera footage material.

\section{DATA}

This section will describe the data used for the prototype. The data is divided into two parts, the data for the crimes and the data for the cameras.

\section{A. Camera data}

The data for the cameras is shown in table I and consists of a name and coordinates representing the exact location of a camera as well as an array of coordinates outlining the catchment area.

\begin{tabular}{lll}
\hline Name & Type & Description \\
\hline Crime code & Integer & Official crime category code \\
Geo-position & Long/Lat & Longitude/latitude coordinates \\
Street & String & Name of the street \\
Street number & String & Street number \\
Postal district & String & Postal district of address \\
Start date & Date & Start date of interval \\
Start time & Time & Start time of interval \\
End date & Date & End date of interval \\
End time & Time & End time of interval \\
\hline
\end{tabular}

TABLE II: Columns in the crime dataset.

\section{B. Crime data}

Table II shows the resulting data extracted from the Excel files provided by the law enforcement. Each crime has a four digit crime code where several codes can be used to group categories of crimes. Each crime also has geo-position marked by longitude and latitude coordinates. Most of the time a crime also has an address with street, street number and postal district. Depending on the type of crime, is has a start date and/or an end date as well as a start time and/or an end time. For example a bicycle theft has most certainly only a start date or an end date but can have a time span in which the bicycle has been stolen. If the victim knows what the time was when he or she got physically abused, there will only be a start date or end date as well as a start time or end time.

\section{WEB-BASED PROTOTYPE}

The prototype utilizes a setup part and a view part. The first step is for the user to choose which crimes to extract from the database. The user adds crime codes one by one or by a grouped selection to a pool of codes. Next step is to select a start date and an end date for the time span of the selected crimes. This data is then used to extract the desired portion of crimes and use in the view part.

The view part uses the selected crimes and displays them as markers on a interactive map. The user can select a span between the start date and end date selected in the setup part. The map then displays the desired crimes. The cameras catchment areas are drawn as colored polygons to easier display where each cameras catchment area is.

\section{A. Database}

The Excel files are first converted to comma separated .csv files. In some entries, one of the dates or timestamps is missing and in those cases the other date or timestamp is used as a placeholder. A MySQL database is then populated with the tabular data to be used by the prototype.

\section{B. Prototype}

The prototype is built with Nodejs ${ }^{1}$ and Express ${ }^{2}$ as backend and JavaScript, HTML, CSS as front end. The map is created using self-hosted map tiles of Sweden. The tiles are

\footnotetext{
${ }^{1}$ https://nodejs.org/en/

${ }^{2} \mathrm{https}$ ://expressjs.com/
} 


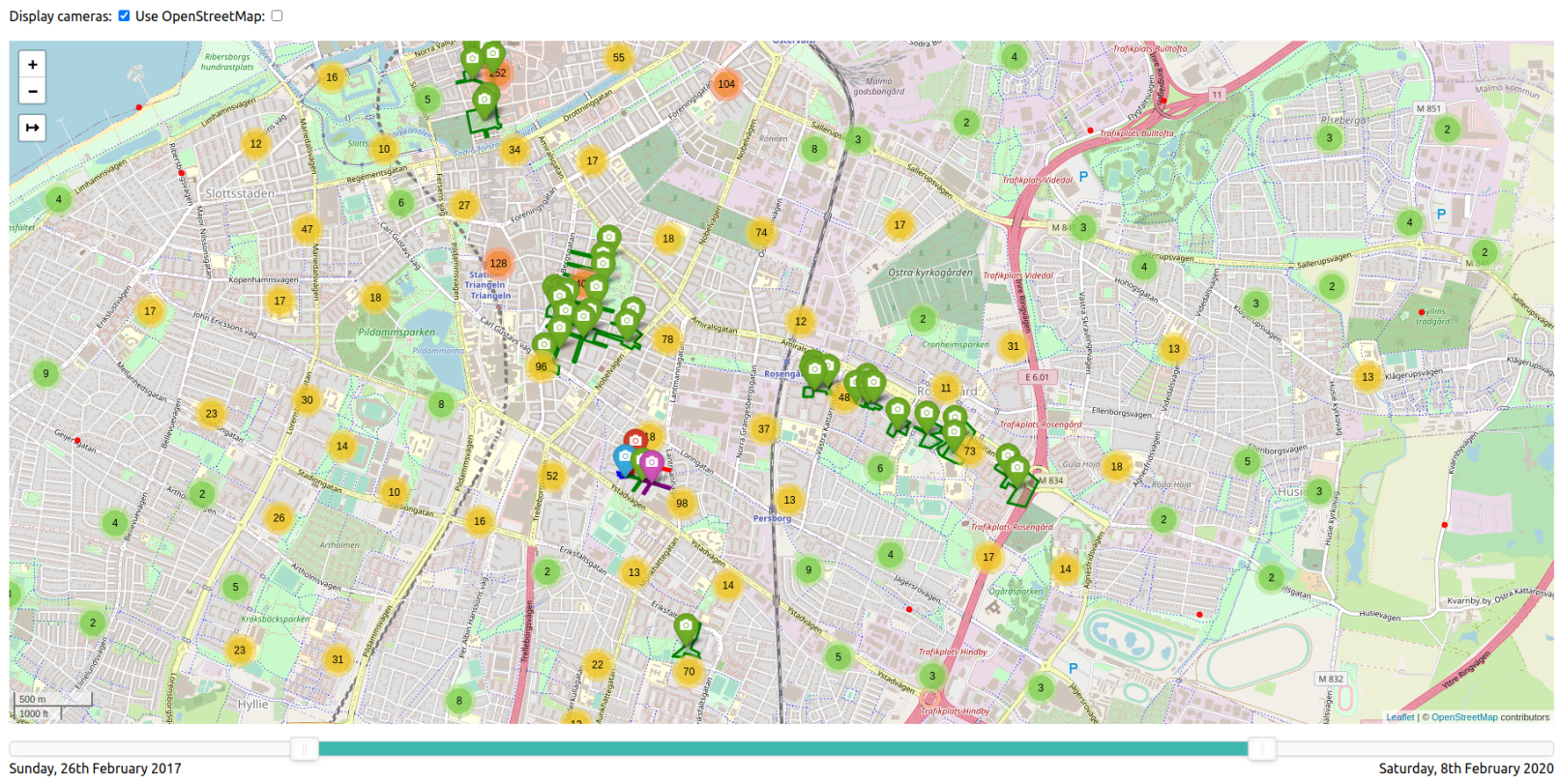

Fig. 1: Overview of all physical abuses 2017-02-26 to 2020-02-08, clustered by occurrences

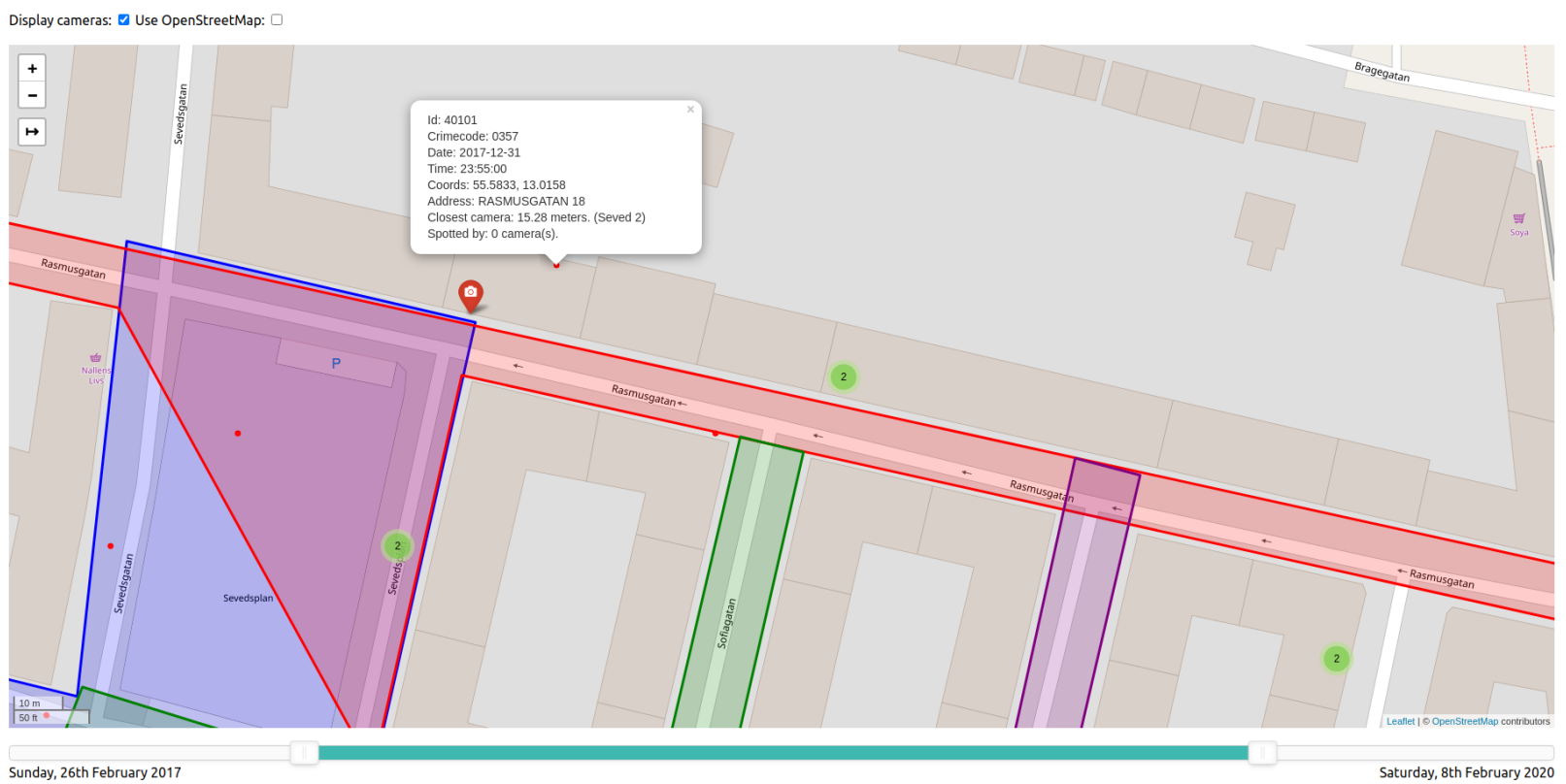

Fig. 2: Zoomed in view that also displays information on a specific crime.

served locally by a Docker container. The functionality for the map is mainly based on the Leaflet ${ }^{3}$ library. A lot of the extra functionality is provided as plugins to Leaflet and are downloaded to be available offline.

The user chooses the crimes to use by crime code as well as start date and end date. When the map is loading, all crimes are fetched from the database and the chosen crimes are extracted and passed on to the map. When dragging the slider all data is removed and new data from within the span is loaded and presented on the map. There is also an option if the map tiles

${ }^{3}$ https://leafletjs.com/ should be provided by the online service OpenStreetMap ${ }^{4}$ instead of the self-hosted alternative.

\section{Visualization}

The screenshot in Figure 1 shows an overview of crimes in crime categories involving various types of physical abuse. The crimes are grouped based on location and are separated based on zoom level. The more the end-user zooms in, the more scattered the groups will become, until individual abuses are shown as red markers. The time slider on the bottom

\footnotetext{
${ }^{4}$ https://www.openstreetmap.org/
} 


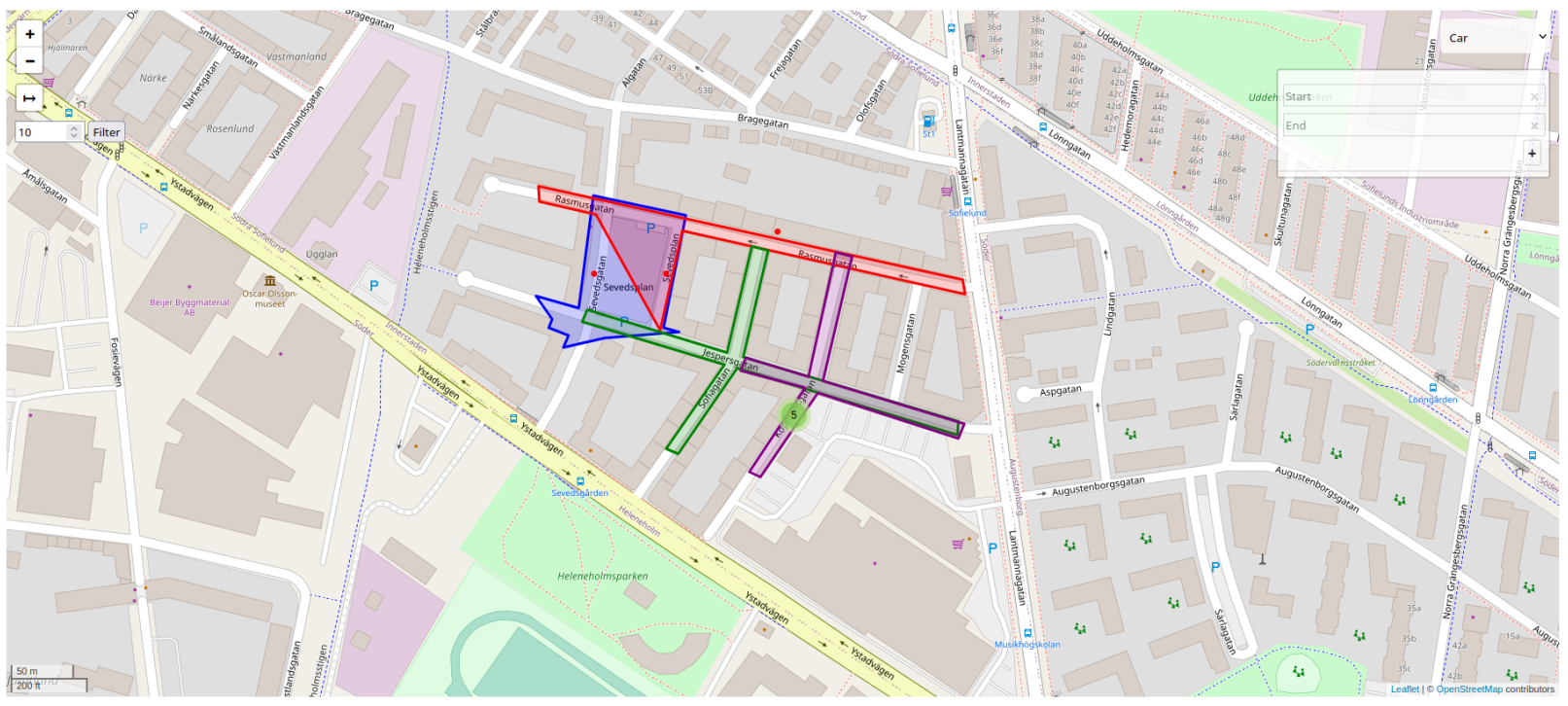

Fig. 3: Filtered to only show assault cases committed within $10 \mathrm{~m}$ of a cameras capture area.

offers an interactive view of crimes within a certain temporal interval, in this case between 2017-02-26 and 2020-02-08. The selected portion in the time slider can be dragged in order to interactively choose more or less crime to be displayed.

Figure 2 displays a more zoomed view of the map. The different colored polygons shows the coverage areas of the individual CCTV cameras. The red camera marker shows the position of a camera and has the red catchment area and so on. Individual crimes are shown as red dots (while clusters of several crimes are shown as green blobs). When clicking on individual crimes an information box is displayed, which shows information about the crime. The information also includes a calculated distance to the closest CCTV camera coverage area boundary, e.g. for the crime in the figure this distance is 15.28 meters to the red area, which belongs to the red CCTV camera coverage areas called "Seved 2".

The information box also includes how many of the CCTV cameras that includes the crime in their coverage areas, i.e. has occurred within one or several cameras catchment areas. To calculate if a coordinate is within a polygon, the plugin PointInPolygon ${ }^{5}$ is used which is based on Dan Sunday's $\mathrm{C}++$ winding number implementation ${ }^{6}$.

\section{CASes}

This section describes three types of crime analysis cases as a way to indicate the prototype tool's practical benefit to the Swedish Police.

\section{A. Case 1: Assault related crimes in 2019}

The first analysis case involves selecting every assaultrelated crimes in Malmö during 2019 that has been committed within 10 meters of any of the CCTV cameras' coverage area. These crimes are of particular interest because there is a

\footnotetext{
${ }^{5}$ https://github.com/hayeswise/Leaflet.PointInPolygon

${ }^{6} \mathrm{http}: / /$ geomalgorithms.com/a03-_inclusion.html
}

high probability of being able to identify the perpetrator. The analyst starts by specifying the relevant crime codes related to assaults, and then specify the date interval, i.e. "From date": 2019-01-01 and "To date": 2019-12-31.

Next, the prototype tool displays the result from the query, and after this the criminal analyst can use the time slider to interactively modify the date range, e.g. to only display crimes a partial date interval such as 2019-06-01 - 2019-0731 . To only display the crimes conducted within 10 meters from the cameras capturing area, the analyst uses the filter function available on the left on the screen in Figure 3. This functionality is of interest because it allows analysts to easily find particular crimes committed where the camera can distinguish different visual features on the perpetrator, such as the color of the clothes or color of a car.

\section{B. Case 2: Drug related crimes}

In the next analysis case the goal for the criminal analysis is find out where most drug related crimes have occurred between 2015 and onward. The prototype have, at the time of writing, access to data up until 2020-12-31. So, the first step is to select all drug related crime codes followed by the date interval. The relevant data is then loaded and displayed in the map view. Next, the analyst can zoom in and out in the map and see the occurred crimes cluster into color-coded circles, see Figure 4a. The orange circles marks that there are a higher amount of crimes occurred on a smaller geographical area, yellow circles marks a slighter lower occurrence and the green marks a relatively low occurrence. When the mouse pointer is placed on a circle, the calculated area is marked on the map. This could be beneficial to see the areas with a high crime rate based on historical data. Together with the drawn capture areas it could also visualize the need for more or fewer cameras in different geographical areas. Based on the selected date interval it could also visualize the crime rate based on different seasons. 


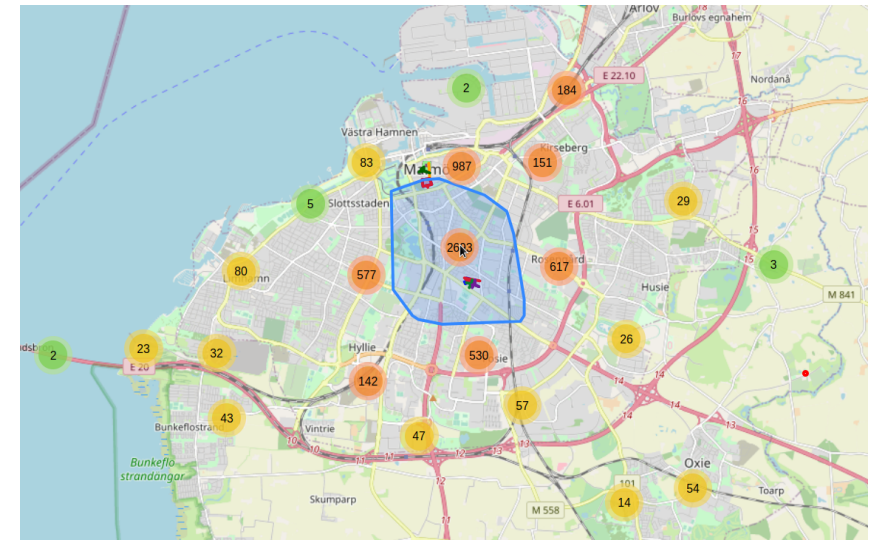

(a)

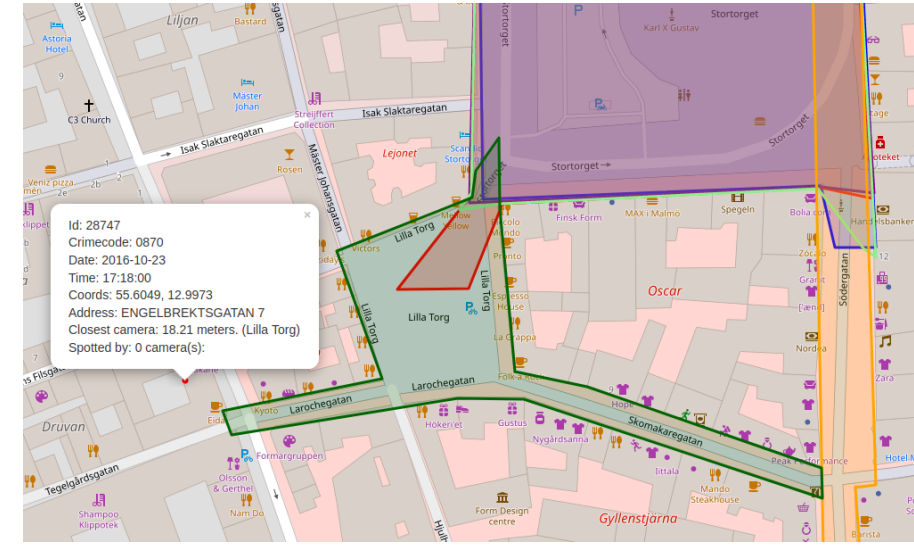

(b)

Fig. 4: (a) Map view of drug related crimes 2015-2020, also including geographical clustering of the crimes. (b) By clicking on a crime on the map, more detailed information about the crime is displayed.

Note that this is an initial clustering functionality and that more capable clustering solutions could be added in the future.

\section{Case 3: Presumed route}

The goal of the last analysis case is to identify CCTV cameras that might have caught the perpetrator(s) after a shop robbery close to the square Lilla Torg in Malmö on the 23rd of October 2016. The law enforcement knows that the perpetrator fled heading east by foot. Thus, the crime analyst starts by choosing the relevant crime code and date. As shown in Figure $4 \mathrm{~b}$ the robbery shows up on the map, and the analyst can click the red dot to get more information about the crime, e.g. the exact address.

The address of the crime is then entered in the box to the right together with a chosen address that east-bound of the crime, which represents where the perpetrator may have moved against. The suggested route of the perpetrator is shown as red lines on the map, see Figure 5. Next, the crime analyst selects "Foot" in the top right drop-down menu, to automatically draw the fastest route by foot. It is also possible to do the analysis when the perpetrator has traveled by car.

Using this route calculation it can be seen that the perpetrator might have been captured by several cameras during this route, e.g. at Lilla Torg (dark green marking) and Södergatan (yellow marking), see Figure 5. If the police have one or more suspects to the crime then the prototype tool can be used together with for instance their home addresses, thus making the tool more relevant. Further development of the tool could include a feature that automatically estimates the time when perpetrators are likely to have passed different CCTV cameras. This would be done by combining the time of the crime with the estimated route and speed of the chosen mode of transport. Such a feature could shorten the process of finding which cameras to investigate, and at which date and time intervals of CCTV camera footage.

\section{DISCUSSION}

The web-based prototype tool can be used for identifying suitable geographical placements for public CCTV cameras through either an iterative manual analysis process, or using an automatic (or semi-automatic) data-driven approach. The later could for instance rely on intelligent models, using machine learning algorithms, for identifying candidate placements for CCTV cameras [7]. Such intelligent models could take into account different aspects related to the crimes. This could for instance involve attempting to maximize the summed crimeharm index for all crimes captured by the cameras, or simply increase the relative weighting of some crime categories, e.g. narcotic-related crimes. Additionally, the framework described in this paper could also be used for determining suitable placement of a set of temporary CCTV cameras throughout the time of a year in order to address the seasonal shift in criminal events in the city of Malmö.

Each camera model has a set of lenses which can provide different quality in the details in the image or video. Some factors that influence the quality are for instance the weather conditions and the time of day. For example if it is daylight, clear weather and the object is close to the camera, the objects face may be visible. If the object is further away, the camera may get a clear video of the suspect's jacket or clothes. The same scenario does not give as clear details if it is recorded at night or if the weather is misty. It would therefore be of interest to the law enforcement officers to extend the current analysis with data on weather conditions. As this would allow the application to provide information on which cameras that may have the best image quality at a certain date, time and location.

In criminal investigations it is interesting for law enforcement officers to determine how suspects have traveled both before and after criminal offences. The web-based prototype can be used as a framework for doing such route-analysis in an semi-automatic fashion. The prototype could make use of functionality that calculates the offender's possible travel routes, based on where the crime has occurred and the type of crime. To further aid in the analysis, the prototype could then give suggestions on what time the offender may have passed in front of different cameras, and thereby aid law enforcement to 


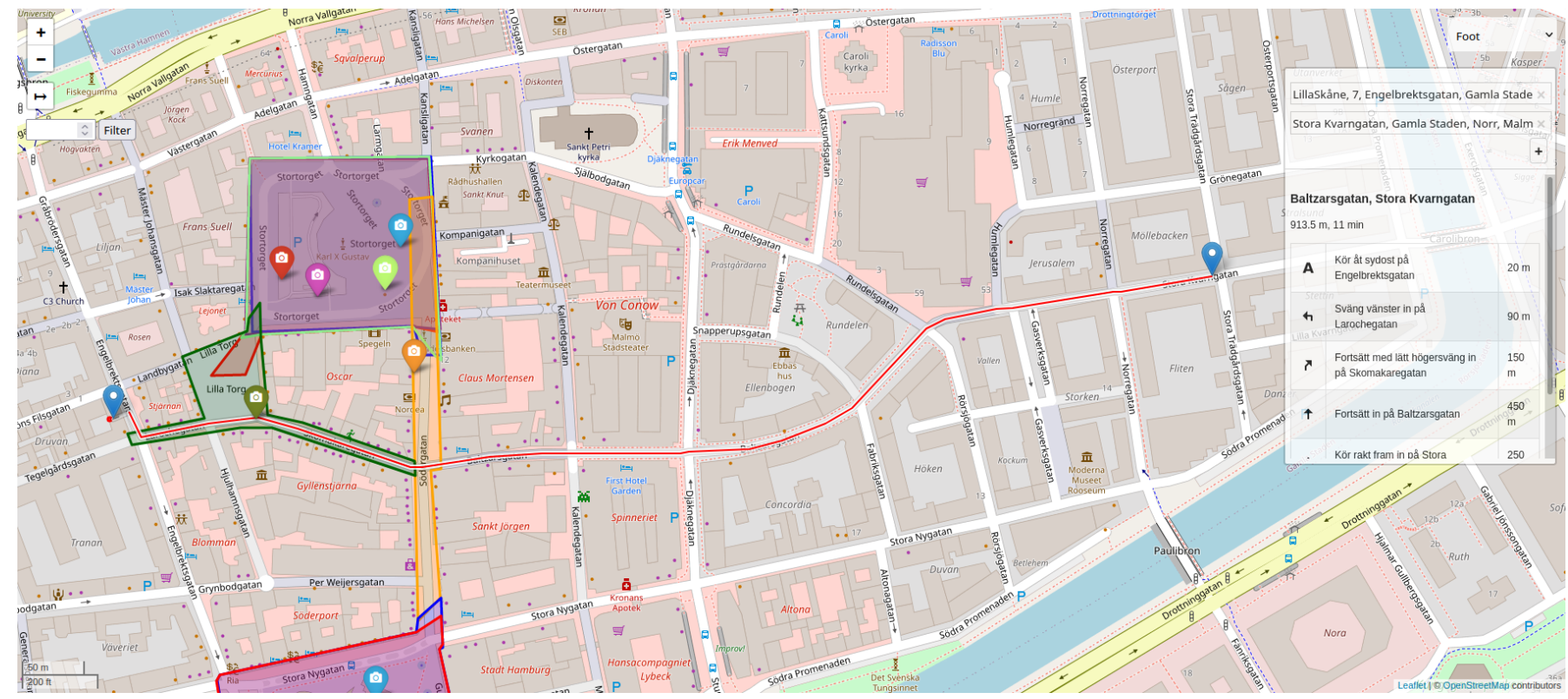

Fig. 5: Investigated route by foot after a crime and the cameras passed.

more efficiently analysing the travel routes used by criminals.

Further, the prototype could benefit from including cameras from external actors. That could extend the catchment areas and make it easier to follow offenders' routes to and from respective crime scenes.

Finally, the web-based prototype also has the benefit of objectively holding all the data regarding the placement of law enforcement-controlled CCTV cameras in the city of Malmö. Prior to using the prototype this data was decentralized and not easily accessible to law enforcement officers. As a result, the prototype can be seen as an additional tool that is provided to Swedish law enforcement. Allowing them much easier access to various analyses (on crime patterns and CCTV cameras) compared to when such analyses had to be conducted in a manual fashion.

\section{CONCLUSIONS \& FUTURE WORK}

This paper presents a web-based prototype for analysis of placement of CCTV cameras with regards to historical crime patterns. The prototype provides a framework for data-driven analyses of CCTV cameras to determine suitable locations for CCTV cameras. The framework could also be used to generate estimates on the amount of detail that is captured by a specific camera given a certain crime event, e.g. taking into account the camera type, distance to the camera, and light/weather condition at the time.

For future work it would be interesting to extend the framework to allow automatic route estimation for suspect(s) moving to/from a crime location, e.g. to estimate the point in time for passing by CCTV cameras. However, this probably would require importing additional cameras from external sources as well, which also is another avenue of future work.

\section{FUNDING}

The research described in this paper was funded by Länsförsäkringar Skåne as part of their five year project for reducing crime and fear in Malmö, and the Skåne region, through applied research on methods for crime prevention.

\section{REFERENCES}

[1] E. L. Piza, "The history, policy implications, and knowledge gaps of the cetv literature: Insights for the development of body-worn video camera research," International Criminal Justice Review, vol. 0, no. 0, p. 1057567718759583, 0. [Online]. Available: https://doi.org/10.1177/1057567718759583

[2] E. L. Piza, B. C. Welsh, D. P. Farrington, and A. L. Thomas, "Cctv surveillance for crime prevention," Criminology \& Public Policy, vol. 18, no. 1, pp. 135-159, 2019. [Online]. Available: https://onlinelibrary.wiley.com/doi/abs/10.1111/1745-9133.12419

[3] D. O. Anderez, E. Kanjo, A. Amnwar, S. Johnson, and D. Lucy, "The rise of technology in crime prevention: Opportunities, challenges and practitioners perspectives," 2021.

[4] M. P. J. Ashby, "The value of cctv surveillance cameras as an investigative tool: An empirical analysis," European Journal on Criminal Policy and Research, vol. 23, pp. 441-459, 2017.

[5] M. Gerell, "Cctv in deprived neighbourhoods - a short-time followup of effects on crime and crime clearance," Nordic Journal of Criminology, vol. 0, no. 0, pp. 1-19, 2020. [Online]. Available: https://doi.org/10.1080/2578983X.2020.1816023

[6] Y. Jung and A. P. Wheeler, "The effect of public surveillance cameras on crime clearance rates," Journal of Experimental Criminology, pp. 1-22, 2021.

[7] J. Kweon and K.-H. Lee, "Proposed placement model for public cctv systems in student safety zones considering surveillance probability on pedestrian streets," Journal of Asian Architecture and Building Engineering, vol. 15, no. 2, pp. 231-238, 2016. [Online]. Available: https://doi.org/10.3130/jaabe.15.231

[8] K. Kim, A. T. Murray, and N. Xiao, "A multiobjective evolutionary algorithm for surveillance sensor placement," Environment and Planning B: Planning and Design, vol. 35, no. 5, pp. 935-948, 2008. [Online]. Available: https://doi.org/10.1068/b33139 